INPLASY

PROTOCOL

To cite: Zeng et al. Th1/Th2 cytokine profiles differentiating tuberculous from malignant pleural effusions: A systematic review and meta-analysis. Inplasy protocol 202210005. doi:

10.37766/inplasy2022.1.0005

Received: 01 January 2022

Published: 01 January 2022

Corresponding author:

Yu Qi

qiyu1987xiaobao@163.com

Author Affiliation:

Xuzhou Central Hospital

Support: No. KC21251. China.

Review Stage at time of this submission: Data analysis.

Conflicts of interest:

None declared.

\section{Th1/Th2 cytokine profiles differentiating tuberculous from malignant pleural effusions: A systematic review and meta-analysis}

Zeng, Y1; Wang, L2; Zhou, H3; Qi, Y4.

Review question / Objective: To clarify which one has a different predominance of Th1 and Th2 immune responses in malignant and tuberculous pleural effusions. We did a metaanalysis of the results published previously to assess the levels of Th1/Th2 cytokines in two types of pleural effusion and evaluated its ability to distinguish TPE from MPE.

Condition being studied: Malignant and tuberculous pleural effusions are the two most common types of exudative pleural effusions, both of which can be seen with the typical accumulation of lymphocytes. Immune responses mediated by either the Th1 or Th2 subset dominate, depending on different types of pleural effusion. Thus, we performed a meta-analysis of all available studies to quantitatively evaluate the levels of Th1/Th2 cytokine profiles in TPE and MPE, as well as to assess the potential diagnostic value of these cytokines in discriminating TPE from MPE.

INPLASY registration number: This protocol was registered with the International Platform of Registered Systematic Review and Meta-Analysis Protocols (INPLASY) on 01 January 2022 and was last updated on 01 January 2022 (registration number INPLASY202210005).

\section{INTRODUCTION}

Review question / Objective: To clarify which one has a different predominance of Th1 and Th2 immune responses in malignant and tuberculous pleural effusions. We did a meta-analysis of the results published previously to assess the levels of Th1/Th2 cytokines in two types of pleural effusion and evaluated its ability to distinguish TPE from MPE.

Condition being studied: Malignant and tuberculous pleural effusions are the two most common types of exudative pleural effusions, both of which can be seen with 
the typical accumulation of lymphocytes. Immune responses mediated by either the Th1 or Th2 subset dominate, depending on different types of pleural effusion. Thus, we performed a meta-analysis of all available studies to quantitatively evaluate the levels of Th1/Th2 cytokine profiles in TPE and MPE, as well as to assess the potential diagnostic value of these cytokines in discriminating TPE from MPE.

\section{METHODS}

Search strategy: Pubmed: ((("Pleural Effusion, Malignant"[Mesh]) OR ((()((Effusion, Malignant Pleural[Title) Abstract]) ) OR (Effusions, Malignant Pleural[Title/Abstract])) OR (Malignant Pleural Effusion[Title/Abstract])) OR (Malignant Pleural Effusions[Title/ Abstract])) OR (Pleural Effusions, Malignant[Title/Abstract]))) OR ((("Pleural Effusion "[Mesh]) OR (( (Effusion, Pleural[Title/Abstract]) OR (Effusions, Pleural[Title/Abstract])) OR (Pleural Effusions[Title/Abstract]))) AND ( $t$ uberculous ) )) A N D ( ( ( ( ( ( ("Interleukin-2"[Mesh]) OR ()(()(()(()(()(Interleukin 2[Title/Abstract]) OR (IL-2[Title/Abstract])) OR (IL2[Title/ Abstract])) OR (TCGF[Title/Abstract])) OR (Interleukine 2[Title/Abstract])) OR (Lymphocyte Mitogenic Factor[Title/ Abstract])) OR (Mitogenic Factor, Lymphocyte[Title/Abstract])) OR (T-Cell Growth Factor[Title/Abstract])) OR (T Cell Growth Factor[Title/Abstract])) OR (T-Cell Stimulating Factor[Title/Abstract])) OR (T Cell Stimulating Factor[Title/Abstract])) OR (Thymocyte Stimulating Factor[Title/ Abstract])) OR (Interleukin II[Title/ Abstract])) OR (Ro 23 6019[Title/Abstract])) OR (RU 49637[Title/Abstract])) OR (RU-49637[Title/Abstract])) OR (RU49637[Title/Abstract]))) OR (("Interferongamma"[Mesh]) OR ( ( ( ( ( (gammaInterferon[Title/Abstract]) OR (Interferon, Immune[Title/Abstract])) OR (Immune Interferon[Title/Abstract])) OR (Type II Interferon[Title/Abstract])) OR (Interferon, Type II[Title/Abstract])) OR (Interferon Type II[Title/Abstract])) OR (Interferon, gamma[Title/Abstract])))) OR (("Tumor Necrosis Factor-alpha"[Mesh]) OR
((()((((Tumor Necrosis Factor alpha[Title) Abstract]) OR (Cachectin[Title/Abstract])) OR (Cachectin-Tumor Necrosis Factor[Title/Abstract])) OR (Cachectin Tumor Necrosis Factor[Title/Abstract])) OR (Tumor Necrosis Factor Ligand Superfamily Member 2[Title/Abstract])) OR (Tumor Necrosis Factor[Title/Abstract])) OR (TNF Superfamily, Member 2[Title/Abstract])) OR (TNFalpha[Title/Abstract])) OR (TNFa Ipha [Title/Abstract]))) ) OR ( ("Interleukin-6"[Mesh]) OR

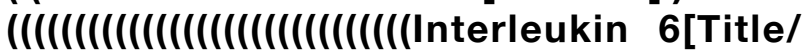
Abstract]) OR (IL6[Title/Abstract])) OR (BCell Stimulatory Factor 2[Title/Abstract])) OR (B-Cell Stimulatory Factor-2[Title/ Abstract])) OR (Differentiation Factor-2, BCell[Title/Abstract])) OR (Differentiation Factor 2, B Cell[Title/Abstract])) OR (B-Cell Differentiation Factor-2[Title/Abstract])) OR (B Cell Differentiation Factor 2[Title/ Abstract])) OR (BSF-2[Title/Abstract])) OR (Hybridoma Growth Factor[Title/Abstract])) OR (Growth Factor, Hybridoma[Title/ Abstract])) OR (IFN-beta 2[Title/Abstract])) OR (Plasmacytoma Growth Factor[Title/ Abstract])) OR (Growth Factor, Plasmacytoma[Title/Abstract])) OR (Hepatocyte-Stimulating Factor[Title/ Abstract])) OR (Hepatocyte Stimulating Factor[Title/Abstract])) OR (MGI-2[Title/ Abstract])) OR (Myeloid DifferentiationInducing Protein[Title/Abstract])) OR (Differentiation-Inducing Protein, Myeloid[Title/Abstract])) OR (Myeloid Differentiation Inducing Protein[Title/ Abstract])) OR (B-Cell Differentiation Factor[Title/Abstract])) OR (B Cell Differentiation Factor[Title/Abstract])) OR (Differentiation Factor, B-Cell[Title/ Abstract])) OR (Differentiation Factor, B Cell[Title/Abstract])) OR (IL-6[Title/ Abstract])) OR (Interferon beta-2[Title/ Abstract])) OR (Interferon beta 2[Title/ Abstract])) OR (beta-2, Interferon[Title/ Abstract])) OR (B Cell Stimulatory Factor-2[Title/Abstract])) OR (B Cell Stimulatory Factor 2[Title/Abstract])))) OR

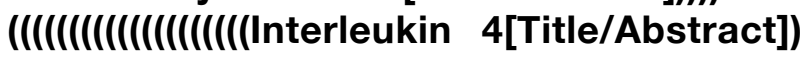
OR (B-Cell Growth Factor-1[Title) Abstract])) OR (B Cell Growth Factor 1[Title/Abstract])) OR (B-Cell Growth Factor-I[Title/Abstract])) OR (B Cell Growth Factor I[Title/Abstract])) OR (B-Cell 
Proliferating Factor[Title/Abstract])) OR (B Cell Proliferating Factor[Title/Abstract])) OR (B-Cell Stimulating Factor-1[Title) Abstract])) OR (B Cell Stimulating Factor 1[Title/Abstract])) OR (B-Cell Stimulatory Factor 1[Title/Abstract])) OR (B-Cell Stimulatory Factor-1[Title/Abstract])) OR (BCGF-1 [Title/Abstract])) OR (Binetrakin [Title/Abstract])) OR (BSF-1[Title/Abstract])) OR (IL-4[Title/ Abstract])) OR (IL4[Title/Abstract])) OR (Mast Cell Growth Factor-2[Title/Abstract])) OR (Mast Cell Growth Factor 2[Title/ Abstract])) OR (B Cell Stimulatory Factor-1[Title/Abstract])) OR (B Cell Stimulatory Factor 1[Title/Abstract]))) OR ( ( "Interleukin-5 " [ M e sh]) OR ()((()(()(Interleukin 5[Title/Abstract]) OR (BCGF-II[Title / A bstract])) OR (Differentiation Factor, Eosinophil[Title/ Abstract])) OR (T-Cell-Replacing Factor[Title/Abstract])) OR (T Cell Replacing Factor[Title/Abstract])) OR (IL-5[Title/Abstract])) OR (IL5[Title/ Abstract])) OR (T-Cell Replacing Factor[Title/Abstract])) OR (Replacing Factor, T-Cell[Title/Abstract])) OR (B-Cell Growth Factor-II[Title/Abstract])) OR (B Cell Growth Factor II[Title/Abstract])) OR (Eosinophil Differentiation Factor[Title/ Abstract])))) OR (("Interleukin-10"[Mesh]) OR (((Interleukin 10[Title/Abstract]) OR (IL10[Title/Abstract])) OR (IL-10[Title/ Abstract])) OR (Cytokine Synthesis Inhibitory Factor[Title/Abstract])))) Filters: from 2000/1/1 - 3000/12/12.

Participant or population: Patients with tuberculous or malignant pleural effusions.

Intervention: Levels of Th1 cytokines.

Comparator: Levels of Th2 cytokines.

Study designs to be included: Case-control studies or cohort studies.

Eligibility criteria: Inclusion criteria: 1) original and human studies; 2) studies with a title or abstract including the terms "tuberculous and malignant pleural effusion" and "cytokines"; 3) studies reporting the pleural effusions levels of TNF-a, IFN- $\gamma$, IL-2, IL-4, IL-5, IL-6, IL-10 in patients; 4) malignant pleural effusion was diagnosed by malignant cells shown in pleural fluid or pleural biopsy; 5) tuberculous pleural effusion was diagnosed by Ziehl-Neelsen stains or positive mycobacteria culture, or pleural biopsy tissue samples were positive; 6) the full text of studies can be found in the database. Exclusion criteria: 1) reviews or metaanalysis; 2) non-human experiments; 3) non-English language literatures.

Information sources: PubMed and Embase databases.

Main outcome(s): Standardized mean differences (SMD).

Data management: With NoteExpress software, duplicate records are firstly eliminated, then deleted records by title or abstract and exclusion criteria, finally, the records that do not meet the main outcomes are removed according to the content of the article.

Quality assessment / Risk of bias analysis: Quality assessment is conducted through the Newcastle-Ottawa Scale(NOS). Begg's test was used in assessing the publication bias.

Strategy of data synthesis: $A \quad P \geq 0.1$ or $12 \leq 50 \%$ was considered as no significant heterogeneity, and the fixed-effects model (FEM) was applied; otherwise, the randomeffects model (REM) was used.

Subgroup analysis: Subgroup analysis based on variables, including country, design, etiology, publishing year, quality scores, and measurement methods.

Sensitivity analysis: Whether the magnitude and direction of the effect size altered substantially by performing a sensitivity analysis by excluding one by one at a time.

Country(ies) involved: China.

Keywords: Th1/Th2 Cytokine, TNF-a, IFN- $\gamma$, IL-6, malignant pleural effusion, tuberculous pleural effusion. 
Contributions of each author:

Author 1 - Yulin Zeng.

Email: yulinzeng725@yahoo.co.jp

Author 2 - Liwei Wang.

Email: 18952170255@163.com

Author 3 - Hai Zhou.

Email: zhouhai339339@163.com

Author 4 - Yu Qi.

Email: qiyu1987xiaobao@163.com 